\title{
Avaliação por tomografia computadorizada helicoidal dos efeitos da expansão rápida da maxila no posicionamento condilar em pacientes com mordida cruzada posterior funcional
}

\begin{abstract}
Edgard Norões Rodrigues da Matta*, Margareth Maria Gomes de Souza**, Eduardo Franzotti de Sant'Anna**, Silvio Chagas da Silva***
\end{abstract}

\section{Resumo}

Objetivo: este estudo clínico avaliou, por meio da tomografia computadorizada helicoidal, os efeitos da expansão rápida da maxila no posicionamento condilar de pacientes com mordida cruzada posterior funcional na fase da dentadura mista. Métodos: dez pacientes com faixa etária entre os 7 anos e 2 meses e os 11 anos e 2 meses - apresentando mordida cruzada posterior funcional, com desvio da linha média de pelo menos $2,5 \mathrm{~mm}$ para o lado do cruzamento - foram tratados com expansão rápida da maxila. Após a obtenção de cortes tomográficos sagitais da articulação temporomandibular, as medidas dos espaços articulares anterior, posterior e superior foram realizadas e o posicionamento relativo do côndilo foi calculado. Utilizandose cortes tomográficos axiais, avaliou-se o posicionamento anteroposterior e transversal dos côndilos em relação às estruturas da base craniana. Resultados: foram identificadas diferenças significativas, antes do tratamento, entre os espaços articulares posteriores, na posição relativa do côndilo e um posicionamento mais anterior e mais próximo ao plano sagital mediano do côndilo do lado não-cruzado. Após o tratamento, não foram evidenciadas diferenças significativas nessas medidas. Conclusões: após a expansão rápida da maxila, um posicionamento mais centralizado dos côndilos nas fossas articulares foi observado, além de uma maior simetria anteroposterior e transversal entre os mesmos.

Palavras-chave: Mordida cruzada funcional. ATM. Tomografia computadorizada.

\section{INTRODUÇÃO}

A expansão rápida da maxila (ERM) é um procedimento terapêutico largamente utilizado em períodos precoces de desenvolvimento oclusal com objetivo de solucionar a deficiência transversal maxilar $2,6,16,21,22,28,30$.
A mordida cruzada posterior unilateral é uma das más oclusões mais frequentes em períodos precoces de desenvolvimento da oclusão, com incidência variando entre $7 \%$ e $23 \%$ da população ${ }^{11,12}$. A mordida cruzada posterior funcional (MCPF) constitui, aproximadamente, de $67 \%$ a $79 \%$ dos casos

* Doutor em Ortodontia pela UFRJ. Professor adjunto de Ortodontia da UFAL.

** Doutores em Ortodontia pela UFRJ. Professores adjuntos de Ortodontia na UFRJ.

*** Professor de Estatística na UFAL. 
de mordida cruzada posterior unilateral ${ }^{12,32}$, e caracteriza-se por um desvio funcional da mandíbula da posição de relação cêntrica para a de máxima intercuspidação habitual ${ }^{1}$. O deslocamento mandibular causa modificação no padrão e intensidade das forças funcionais aplicadas na mandíbula e na articulação temporomandibular ${ }^{14}$. Em pacientes que apresentam mordida cruzada funcional, têm sido relatadas, na literatura, alterações musculares decorrentes da atividade assimétrica entre os músculos homólogos e a ocorrência de possível alteração na relação geométrica do côndilo mandibular na fossa articular $8,20,22$.

Na maioria dos casos, essa discrepância oclusal é consequência da deficiência transversal do arco superior, que encontra-se atrésico, em razão, geralmente, de obstrução das vias aéreas superiores, devido ao tecido adenoidiano hipertrofiado ou alergias nasais, com consequente respiração bucal ${ }^{4,17}$, e também hábitos de sucção de chupeta e/ou dedo $0^{9,15,18}$.

A expansão maxilar é considerada o tratamento de escolha para as mordidas cruzadas funcionais porque soluciona a deficiência transversal maxilar, eliminando o desvio funcional da mandíbula ${ }^{22}$. Diferentes métodos têm sido utilizados para expandir o arco maxilar constricto, classificando-se em diferentes mecanismos que produzem expansão lenta, semirrápida ou rápida ${ }^{27}$. A ERM é um procedimento extremamente vantajoso no tratamento da deficiência maxilar transversal ${ }^{3}$.

A avaliação da articulação temporomandibular (ATM), nos casos de MCPF, sempre foi realizada com a utilização de radiografia transcraniana e tomografia linear. $\mathrm{O}$ uso de radiografia transcraniana apresenta grande potencial de erro metodológico, devido à qualidade da imagem ficar comprometida e aos efeitos de projeção ${ }^{19}$. Segundo Knoernschild, Aquilino e Ruprecht ${ }^{10}$, as técnicas transcranianas, incluindo a corrigida, não reproduzem com precisão a anatomia do espaço articular, sendo isso somente possível com a tomografia linear. Entretanto, Lam, Sadowski e Omerza ${ }^{13}$ concluíram que a utilização de tomografia linear corrigida para a mensuração dos espaços articulares da ATM deve ter limitado valor de diagnóstico, sendo incapaz de demonstrar diferenças na posição condilar.

Atualmente, avanços tecnológicos têm contribuído para aprimorar as técnicas de diagnóstico, permitindo uma avaliação minuciosa e precisa das estruturas anatômicas. A tomografia computadorizada (TC) constitui um dos grandes avanços da atualidade na área de imagem, obtendo-se com mais segurança a avaliação da ATM e fornecendo informações dos espaços articulares ${ }^{31}$. Também permite a eliminação da sobreposição de estruturas vizinhas. Sendo assim, a imagem obtida representa um corte nítido da área em estudo ${ }^{25}$, além da excelente precisão da TC colaborar para resultados confiáveis ${ }^{5}$. Estudos recentes têm utilizado a TC com cone beam para avaliar os efeitos esqueléticos após a $\mathrm{ERM}^{7}$, e a TC convencional tem sido utilizada para avaliar alterações na posição condilar ${ }^{34}$.

Dessa forma, o presente trabalho teve como objetivo avaliar, por meio de imagens de TC, os efeitos da ERM no posicionamento condilar em pacientes apresentando MCPF.

\section{MATERIAL E MÉTODOS}

$\mathrm{O}$ protocolo de pesquisa desse trabalho foi aprovado pelo Comitê de Ética em Pesquisa da Universidade Federal de Alagoas, com registro de processo $n^{\circ}$ 0087902004-54. Os responsáveis pelos pacientes selecionados para a amostra, após serem orientados e terem todas as dúvidas esclarecidas, assinaram um Termo de Consentimento Livre e Esclarecido.

A amostra consistiu de 10 pacientes (5 do gênero masculino e 5 do gênero feminino) apresentando MCPF com idades variando de 7 anos e 2 meses a 11 anos e 2 meses. A mordida foi considerada MCPF quando o paciente, em máxima intercuspidação habitual, apresentava sobreposição unilateral das cúspides vestibulares dos dentes posteriores inferiores às cúspides vestibulares dos dentes superiores ${ }^{32}$, além de desvio da linha média 
dentária inferior para o lado do cruzamento ${ }^{8,13,26}$. Na mandíbula, quando guiada para relação cêntrica, o cruzamento transformava-se em mordida de topo bilateral com coincidência das linhas médias ${ }^{29}$. Sendo assim, todos os pacientes tinham má oclusão transversal com algum grau de constrição da maxila. A constrição maxilar foi determinada pela avaliação da forma do arco superior e pelo relacionamento entre os arcos dentários superior e inferior.

Todos os pacientes enquadravam-se nos seguintes critérios de inclusão: ausência de cárie interproximal extensa ou perda dentária na região posterior, bom relacionamento das bases ósseas e dos arcos dentários no sentido vertical e anteroposterior, ausência de sinais ou sintomas de disfunção temporomandibular, e presença de desvio da linha média de, pelo menos, $2,5 \mathrm{~mm}$ para o lado do cruzamento. Os pacientes não tinham sido submetidos a tratamento ortodôntico prévio, não apresentavam mordida cruzada anterior, tendência à Classe III, histórico de trauma na região facial ou dentária, nem anomalias de crescimento clinicamente evidentes.

Os pacientes foram submetidos a exames com TC antes e após a ERM. As tomografias de controle pós-expansão somente foram realizadas após o período de contenção, quando o aparelho expansor foi removido e a foi observada clinicamente a presença de uma situação de estabilidade oclusal.

Para a obtenção das imagens, utilizou-se um equipamento de Tomografia Computadorizada, modelo CT/e (General Electric Medical Systems, Tóquio, Japão), com 120kV e 120mA, por meio da técnica helicoidal com cortes de $1 \mathrm{~mm}$ de espessura e $1 \mathrm{~mm}$ de intervalo no plano axial (Fig. 1A). Em seguida, realizou-se a reformatação ou reconstrução, que consiste na obtenção de imagens no plano sagital a partir da realização do exame com incidência da radiação no plano axial, uma vez que o exame sagital direto é de realização complexa, devido à dificuldade de posicionamento do paciente. As imagens selecionadas foram processadas no equipamento Advantage Windows I (General Eletric).
As imagens de TC foram obtidas com os pacientes em máxima intercuspidação habitual e suas cabeças orientadas com o plano horizontal de Frankfurt e o plano médio sagital perpendiculares ao solo, tendo sido utilizado um posicionador de cabeça acoplado à mesa do tomógrafo para padronizar esse posicionamento. $\mathrm{O}$ posicionador de cabeça apresentava dois posicionadores auriculares, com controle de movimento no plano horizontal para permitir aproximação e distanciamento do meato acústico do paciente, e também no plano vertical (Fig. 1B). Como mais um parâmetro para padronização, utilizou-se o próprio sistema de laser do equipamento, que emite feixe de luz longitudinal passando através do centro da glabela e filtrum, e outro feixe de luz transversal unindo os cantos externos dos olhos. Para evitar a formação de artefato de imagem, após a padronização do posicionamento da cabeça do paciente, a fixação da mesma era realizada com elástico contendo velcro na testa do paciente e na região do mento (Fig. 1C). Após o posicionamento do paciente, um pré-escaneamento era realizado e, na sala da estação de trabalho, anexa à sala de exame, a avaliação do posicionamento do paciente era realizada e, em seguida, definida a área da execução dos cortes tomográficos (Fig. 2A, B).

Para a realização da ERM utilizou-se o aparelho disjuntor do tipo Haas modificado com protocolo de ativação de $1 / 4$ de volta pela manhã e $1 / 4$ de volta à tarde, até que se conseguisse uma sobrecorreção, em média, de $2 \mathrm{~mm}$. Após o período ativo de expansão, o parafuso foi estabilizado e o aparelho disjuntor foi mantido por 3 meses de contenção.

Após a obtenção e seleção das imagens, as medidas dos espaços articulares anterior, superior e posterior foram realizadas na imagem impressa em filme radiográfico, com a utilização de traçados das estruturas em estudo, seguindo a metodologia já utilizada por Vitral et al. ${ }^{33}$ Para a avaliação do posicionamento anteroposterior e transversal dos côndilos mandibulares em relação às estruturas da base craniana, utilizou-se a metodologia já 


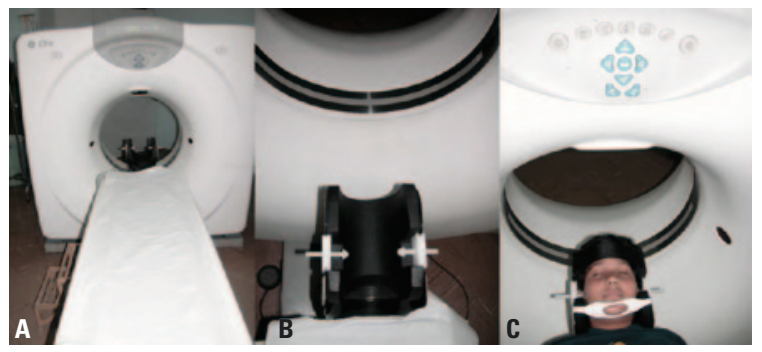

FIGURA 1 - A) Tomógrafo utilizado para obtenção das imagens; B) posicionador usado na padronização do posicionamento da cabeça e C) posicionamento do paciente para obtenção das imagens.

divulgada por Pirttiniemi et al. ${ }^{23}$

As dimensões das imagens nas tomografias computadorizadas não correspondem ao tamanho real das mesmas, dessa forma, foi incorporada uma escala com um segmento de reta de $10 \mathrm{~mm}$ impresso em cada figura para permitir a conversão das medidas.

Para a realização dos traçados, utilizou-se papel acetato transparente Ultraphan ${ }^{\circledR}$, marca UNITEK, e lápis HB 0,5mm. Para as medidas, foi usado paquímetro eletrônico digital da marca Starret (Itu/ SP), modelo 727, e as seguintes medições foram realizadas, por um único operador, nas imagens do corte sagital (Fig. 3A, B):

- Espaço articular anterior (EAA): representado pela menor distância entre o ponto mais anterior do côndilo e a parede posterior do tubérculo articular.

- Espaço articular superior (EAS): medição da menor distância entre o ponto mais superior do côndilo e o ponto mais superior da fossa mandibular.

- Espaço articular posterior (EAP): mensuração da menor distância entre o ponto mais posterior do côndilo e a parede posterior da fossa mandibular.

Para avaliar a posição relativa de cada côndilo, utilizou-se a fórmula preconizada por Pullinger et al. ${ }^{24}$, que se expressa matematicamente assim: espaço articular posterior menos espaço articular anterior dividido pela soma do espaço articular anterior e posterior, expresso como percentual.

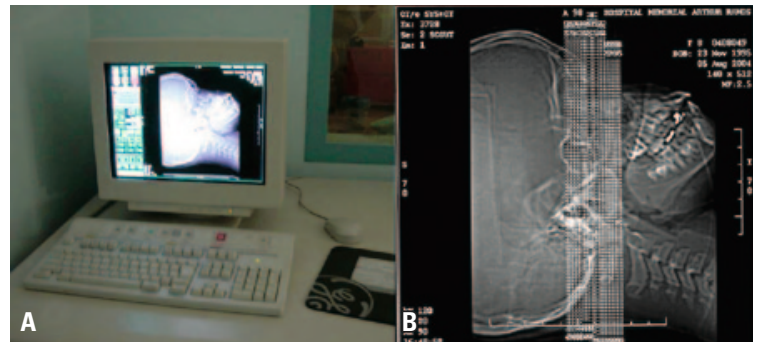

FIGURA 2 - A) Estação de trabalho onde as imagens foram avaliadas e B) fotografia de imagem do pré-escaneamento.

A localização do côndilo centralizado na fossa foi indicada pelo valor zero; um valor percentual negativo indicou uma localização posterior e um valor percentual positivo um deslocamento anterior.

Sobre as imagens no plano axial, impressas em filme radiográfico, foi colocado o mesmo tipo de papel já mencionado, e duas linhas de referência foram traçadas, com objetivo de avaliar o posicionamento dos côndilos em relação à base craniana, seguindo a metodologia utilizada por Pirttiniemi et al. ${ }^{23}$ (Fig. 4A, B):

- Linha de referência sagital mediana (LRSM), que passa através do plano médio sagital do crânio.

- Linha de referência transversal (LRT), que é perpendicular à linha de referência sagital mediana e tangencia o limite posterior do clivo.

A avaliação do posicionamento anteroposterior dos côndilos foi realizada medindo-se a distância, em milímetros, do centro geométrico do côndilo à LRT; e a avaliação do posicionamento transversal foi executada medindo-se a distância, também em milímetros, do centro geométrico do côndilo à LRSM, sendo utilizado um paquímetro eletrônico digital (modelo 727, Starrett) para executar as referidas medidas.

Os valores médios encontrados para cada medida estudada foram submetidos ao tratamento estatístico, tendo sido aplicado o teste dos postos assinalados de Wilcoxon, para avaliar se existia diferença significativa entre o posicionamento dos côndilos nos lados cruzado e não-cruzado, antes e após o tratamento. 


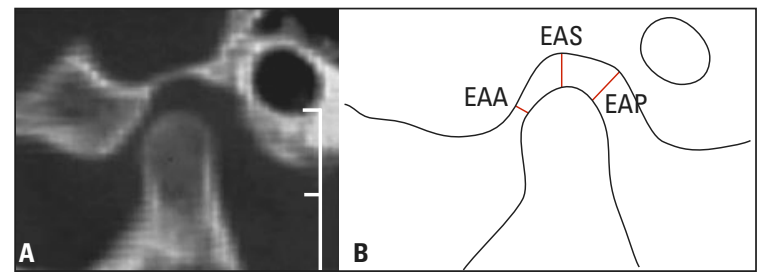

FIGURA 3 - A) Fotografia de imagem tomográfica sagital e B) desenho esquemático das medidas do espaço articular anterior (EAA), espaço articular superior (EAS) e espaço articular posterior (EAP).

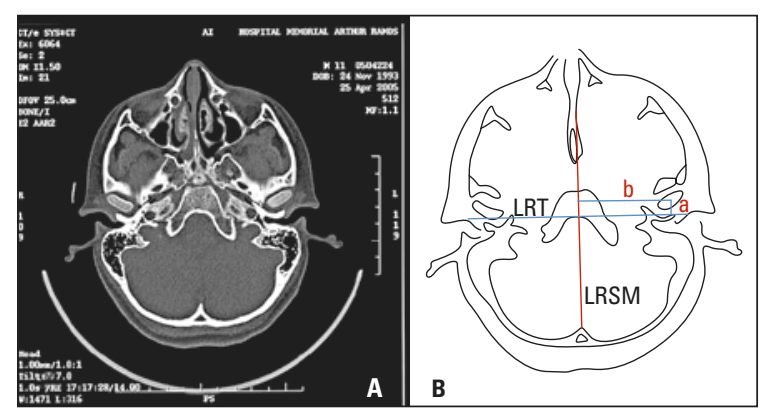

FIGURA 4 - Fotografia (A) e traçado (B) da imagem tomográfica axial: medidas do posicionamento anteroposterior (a) e transversal (b) do côndilo; linha de referência sagital mediana (LRSM) e linha de referência transversal (LRT).

\section{RESULTADOS}

A tabela 1 apresenta a média e o desvio-padrão para cada variável avaliada e o resultado da análise estatística comparando-se o lado não-cruzado (NC) com o lado cruzado (C), antes e após o tratamento.

$\mathrm{Na}$ fase pré-tratamento, os resultados encontrados nesse trabalho evidenciaram diferenças estatisticamente significativas no espaço articular posterior, na posição relativa do côndilo, na posição anteroposterior e na posição transversal dos côndilos em relação às estruturas da base do crânio.

$\mathrm{Na}$ avaliação do espaço articular posterior, maior espaço articular foi encontrado no lado NC, média de $3,26 \mathrm{~mm}$, do que no lado $C$, média de $2,82 \mathrm{~mm}(\mathrm{p}=0,047)$. A posição relativa do côndilo apresentou valores médios para os lados $\mathrm{NC}$ e C de $40,5 \%$ e $33,09 \%$, respectivamente ( $p=0,047)$.

Os valores médios para o posicionamento anteroposterior do côndilo em relação às estruturas da base craniana foram de $8,57 \mathrm{~mm}$ para lado NC e de $7,17 \mathrm{~mm}$ para lado $C(p=0,009)$, enquanto no posicionamento transversal foram encontradas médias de $45,42 \mathrm{~mm}$ e $46,96 \mathrm{~mm}$ para os lados NC e C $(\mathrm{p}=0,005)$, respectivamente.

$\mathrm{Na}$ fase pós-tratamento, diferença estatisticamente significativa foi observada somente no espaço articular superior, apresentando valor médio de $1,98 \mathrm{~mm}$ no lado $\mathrm{NC}$ e $2,24 \mathrm{~mm}$ no lado C (p $=0,012$ ).

Na tabela 2, encontram-se a média e o desviopadrão para cada variável avaliada e o resultado da análise estatística comparando-se as modificações ocorridas em cada lado separadamente, antes e após o tratamento.

No lado NC, diferenças estatisticamente significativas entre as fases pré e pós-tratamento foram encontradas no espaço articular posterior, na posição relativa do côndilo e na posição anteroposterior dos côndilos em relação às estruturas da base do crânio.

O espaço articular posterior apresentou média pré-tratamento de $3,26 \mathrm{~mm}$ e pós-tratamento de $2,46 \mathrm{~mm}(\mathrm{p}=0,017)$. Na avaliação da posição relativa do côndilo, foram encontrados valores médios de $40,5 \%$ e $26,75 \%$ para as fases pré e pós-tratamento, respectivamente $(\mathrm{p}=0,009)$. Os valores médios para o posicionamento anteroposterior do côndilo em relação às estruturas da base craniana foram de 8,57mm antes da ERM e 7,21 mm após a expansão $(\mathrm{p}=0,005)$.

No lado $C$, diferenças estatisticamente significativas entre as fases pré e pós-tratamento foram encontradas somente na posição relativa do côndilo e na posição transversal dos côndilos em relação às estruturas da base do crânio.

$\mathrm{Na}$ avaliação da posição relativa do côndilo, a diferença entre as médias encontradas nas fases pré-tratamento $(33,09 \%)$ e pós-tratamento $(25,2 \%)$ foi significativa, com $\mathrm{p}$ valor de 0,022 . A posição transversal do côndilo apresentou valor médio de 46,96mm na fase antes da ERM, e de $46,33 \mathrm{~mm}$ após a expansão $(\mathrm{p}=0,021)$. 
TABELA 1 - Média, desvio-padrão e resultado da análise estatística das medidas dos espaços articulares, posição relativa do côndilo e posicionamento dos côndilos em relação às estruturas da base do crânio (pré e pós-tratamento).

\begin{tabular}{|c|c|c|c|c|c|c|c|c|c|c|}
\hline & \multicolumn{5}{|c|}{ PRÉ-TRATAMENTO } & \multicolumn{5}{|c|}{ PÓS-TRATAMENTO } \\
\hline & \multicolumn{2}{|c|}{ lado NC } & \multicolumn{2}{|c|}{ lado C } & \multirow{2}{*}{$\mathbf{p}$} & \multicolumn{2}{|c|}{ lado NC } & \multicolumn{2}{|c|}{ lado C } & \multirow{2}{*}{$\mathbf{p}$} \\
\hline & média & d.p. & média & d.p. & & média & d.p. & média & d.p. & \\
\hline $\mathrm{EAS}(\mathrm{mm})$ & 2,46 & 0,69 & 2,29 & 0,96 & 0,221 & 1,98 & 0,87 & 2,24 & 0,81 & $0,012^{*}$ \\
\hline $\mathrm{EAA}(\mathrm{mm})$ & 1,31 & 0,32 & 1,38 & 0,39 & 0,644 & 1,25 & 0,2 & 1,36 & 0,33 & 0,066 \\
\hline $\operatorname{EAP}(\mathrm{mm})$ & 3,26 & 0,97 & 2,82 & 0,75 & $0,047^{*}$ & 2,46 & 1,06 & 2,48 & 1 & 0,721 \\
\hline PRC (\%) & 40,5 & 15,61 & 33,09 & 18,14 & $0,047^{*}$ & 26,75 & 21,74 & 25,2 & 25,37 & 0,721 \\
\hline $\begin{array}{l}\text { Pos. AP - } \\
\text { BC (mm) }\end{array}$ & 8,57 & 3,5 & 7,17 & 2,58 & $0,009 * *$ & 7,21 & 2,51 & 7,06 & 2,68 & 0,386 \\
\hline $\begin{array}{l}\text { Pos. transv. - } \\
\text { BC }(\mathrm{mm})\end{array}$ & 45,42 & 2,78 & 46,96 & 3,43 & $0,005^{* *}$ & 45,84 & 2,48 & 46,33 & 3,3 & 0,333 \\
\hline
\end{tabular}

* Significativo ao nível de 5\%, ** Significativo ao nível de 1\%. Lado NC = lado não-cruzado, lado C = lado cruzado.

EAS = espaço articular superior, EAA = espaço articular anterior, EAP = espaço articular posterior, $\mathbf{P R C}=$ posição relativa do côndilo,

Pos. AP-BC = posição anteroposterior-base craniana, Pos. transv.-BC = posição transversal-base craniana, $\mathbf{d}$.p. $=$ desvio-padrão

TABELA 2 - Média, desvio-padrão e resultado da análise estatística das medidas dos espaços articulares, posição relativa do côndilo e posicionamento dos côndilos em relação às estruturas da base do crânio, dos lados não-cruzado e cruzado (pré e pós-tratamento).

\begin{tabular}{|c|c|c|c|c|c|c|c|c|c|c|}
\hline & \multicolumn{5}{|c|}{ LADO NÃO-CRUZADO } & \multicolumn{5}{|c|}{ LADO CRUZADO } \\
\hline & \multicolumn{2}{|c|}{ pré-tratamento } & \multicolumn{2}{|c|}{ pós-tratamento } & \multirow{2}{*}{$\mathbf{p}$} & \multicolumn{2}{|c|}{ pré-tratamento } & \multicolumn{2}{|c|}{ pós-tratamento } & \multirow[t]{2}{*}{$\mathbf{p}$} \\
\hline & média & d.p. & média & d.p. & & média & d.p. & média & d.p. & \\
\hline $\mathrm{EAS}(\mathrm{mm})$ & 2,46 & 0,69 & 1,98 & 0,87 & 0,139 & 2,29 & 0,96 & 2,24 & 0,81 & 0,575 \\
\hline $\mathrm{EAA}(\mathrm{mm})$ & 1,31 & 0,32 & 1,25 & 0,2 & 0,646 & 1,38 & 0,39 & 1,36 & 0,33 & 0,284 \\
\hline $\operatorname{EAP}(\mathrm{mm})$ & 3,26 & 0,97 & 2,46 & 1,06 & $0,017^{*}$ & 2,82 & 0,75 & 2,48 & 1 & 0,185 \\
\hline PRC (\%) & 40,5 & 15,61 & 26,75 & 21,74 & $0,009 * *$ & 33,09 & 18,14 & 25,2 & 25,37 & $0,022^{*}$ \\
\hline $\begin{array}{l}\text { Pos. AP- } \\
\text { BC (mm) }\end{array}$ & 8,57 & 3,5 & 7,21 & 2,51 & $0,005^{* *}$ & 7,17 & 2,58 & 7,06 & 2,68 & 0,799 \\
\hline $\begin{array}{l}\text { Pos. Transv.- } \\
\text { BC }(\mathrm{mm})\end{array}$ & 45,42 & 2,78 & 45,84 & 2,48 & 0,093 & 46,96 & 3,43 & 46,33 & 3,3 & $0,021^{*}$ \\
\hline
\end{tabular}

* Significativo ao nível de 5\%, ** Significativo ao nível de $1 \%$.

EAS = espaço articular superior, EAA = espaço articular anterior, EAP = espaço articular posterior, $\mathbf{P R C}=$ posição relativa do côndilo,

Pos. AP-BC = posição anteroposterior-base craniana, Pos. transv.-BC = posição transversal-base craniana, d.p. = desvio-padrão.

\section{DISCUSSÃO}

A ERM é um procedimento rotineiramente usado em pacientes que apresentam MCPF para solucionar a constrição da maxila nas dentaduras decídua, mista e permanente. O propósito desse estudo foi comparar o posicionamento condilar na fossa mandibular e em relação às estruturas da base craniana, antes e após a ERM. No plano vertical, a investigação foi realizada por meio da avaliação do espaço articular superior. No plano sagital, utilizou-se os espaços articulares anterior e posterior, a posição relativa do côndilo e a análise das modificações anteroposteriores em relação às estruturas de referência craniana, e no plano transversal também em relação às estruturas da base craniana. 
$\mathrm{Na}$ fase pré-tratamento, as diferenças estatisticamente significativas observadas no espaço articular posterior, na posição relativa do côndilo e na posição anteroposterior na base craniana demonstraram um posicionamento do côndilo do lado NC mais anterior na fossa mandibular em relação ao lado C. Essa observação está em concordância com os resultados de Myers et al..$^{20}$ e Hesse et al. ${ }^{8}$ e, consequentemente, discordam de Lam, Sadowski e Omerza ${ }^{13}$. Diferença significativa também foi encontrada na posição transversal do côndilo, estando o côndilo do lado NC mais próximo à linha de referência sagital mediana. Após a ERM, não foram observadas diferenças estatisticamente significativas nessas variáveis, evidenciando a influência que esse procedimento terapêutico teve sobre o posicionamento condilar tanto sagital quanto transversal. Esses achados estão em acordo com os estudos de Myers et al. ${ }^{20}$, Hesse et al. ${ }^{8} \mathrm{e}$ Pinto et al. ${ }^{22}$

$\mathrm{Na}$ fase pós-tratamento, diferença estatística significativa foi encontrada somente no espaço articular superior. Antes do tratamento, o espaço articular superior do lado não-cruzado apresentava-se maior do que no lado cruzado, embora as diferenças não fossem significativas. Após a ERM, situação inversa ocorreu, estando o lado que apresentava a mordida cruzada com espaço maior que o oposto, tendo essa observação sido relatada também por Hesse et al. ${ }^{8}$

A avaliação das alterações ocorridas em cada côndilo separadamente fornece dados com respeito à modificação induzida pela ERM em cada lado. No lado NC, as diferenças estatísticas foram significativas no espaço articular posterior, na posição relativa do côndilo e na posição anteroposterior em relação à base craniana.

Uma tendência ao posicionamento mais posterior do côndilo do lado NC, após a ERM, foi evidenciada pela redução significativa do espaço articular posterior de $3,26 \mathrm{~mm}$, no pré-tratamento, para $2,46 \mathrm{~mm}$, após o tratamento $(\mathrm{p}=0,017)$, bem como, também, pela avaliação da posição relativa do côndilo, com diminuição de 40,5\% para $26,75 \%(p=0,009)$, permitindo, dessa forma, um posicionamemto mais centralizado. Essa observação também foi relata por Hesse et al. ${ }^{8}$ Em relação às estruturas de referência craniana, a redução na posição anteroposterior de $8,57 \mathrm{~mm}$ para $7,21 \mathrm{~mm}$ ( $\mathrm{p}=0,005)$ também permite deduzir a ocorrência do deslocamento do côndilo do lado NC para posterior. No plano transversal, observou-se um aumento na média de $45,42 \mathrm{~mm}$ (pré-tratamento) para $45,84 \mathrm{~mm}$ (pós-tratamento), denotando um distanciamento do côndilo do lado NC da linha de referência sagital mediana, embora essa alteração não seja estatisticamente significativa.

No lado C, diferenças significativas foram encontradas somente na posição relativa do côndilo e na posição transversal em relação às estruturas cranianas. A redução da posição relativa do côndilo de 33,09\% (pré-tratamento) para 25,20\% (póstratamento) evidenciou uma melhora no posicionamento relativo do côndilo em função da ERM. $\mathrm{Na}$ posição transversal do côndilo do lado $\mathrm{C}$ em relação às estruturas do crânio observou-se uma redução média de 46,96mm (pré-tratamento) para 46,33 (pós-expansão), demonstrando um posicionamento mais próximo da linha de referência sagital mediana.

Os dados encontrados nesse trabalho permitiram deduzir que as maiores mudanças foram verificadas no côndilo do lado NC, que apresentava, antes da ERM, maiores alterações, em razão da mordida cruzada posterior funcional. Observação também nesse sentido é referenciada por Myers et al. ${ }^{20}$ e Hesse et al. ${ }^{8}$

A presença de posicionamento não-centralizado dos côndilos nas fossas mandibulares e a presença de uma assimetria entre os côndilos, em relação às estruturas de referência craniana, foram constatadas nos pacientes com mordida cruzada posterior funcional. A ERM, como opção de tratamento dessa má oclusão, promoveu alterações na localização dos côndilos que permitiram um melhor posicionamento desses. 


\section{CONCLUSÃO}

O tratamento da MCPF por meio da ERM influenciou a localização dos côndilos nas fossas mandibulares, permitindo um posicionamento mais centralizado dos mesmos. Em relação às estruturas da base craniana, promoveu maior simetria anteroposterior e transversal entre os processos condilares.

\title{
Evaluation with helicoidal computed tomography of rapid maxillary expansion effects in the condylar position of patients with functional posterior crossbite
}

\begin{abstract}
Aim: This clinical study evaluated, through helicoidal computed tomography (CT), the effects of rapid maxillary expansion in the condylar position of patients with functional posterior crossbite in mixed dentition stage. Methods: Ten patients aged between 7 years and 2 months and 11 years and 2 months were selected. This patients which presented functional unilateral posterior crossbite with mandibular midline deviation of at least $2.5 \mathrm{~mm}$ to the crossbite side were submitted to rapid maxillary expansion. After obtaining sagittal CT slices of the temporomandibular joints, measurements of the anterior, posterior and superior joint spaces were carried out and the relative positioning of the condyle was calculated. Axial slices of the temporomandibular joint were also used to evaluate the anteroposterior and transversal position of the condyles in relation to the cranial base structures. Results: Before treatment, significant differences were observed in the posterior joint spaces, in the relative positioning of the condyle and with the non-crossbite side condyle displaced more anteriorly and medially in relation to midsagittal line. After treatment there was no statistically significant difference. Conclusion: After the rapid maxillary expansion, a more centralized position of the condyles in the joint cavities was observed, and a greater anteroposterior and transverse symmetry between them.
\end{abstract}

Keywords: Functional posterior crossbite. TMJ. Computed tomography.

\section{REFERÊNCIAS}

1. AHLGREN, J.; POSSELT, U. Need of functional analysis and selective grinding in orthodontics: A clinical and electromyographic study. Acta Odontol. Scand., London, v. 21, p. 187-226, June 1963.

2. ARAT, F. E. et al. Muscular and condylar response to rapid maxillary expansion. Part 2: Magnetic resonance imaging study of temporomandibular joint. Am. J. Orthod. Dentofacial Orthop., St. Louis, v. 133, no. 6, p. 823-829, June 2008.

3. BICAKCl, A. A. et al. Nasal airway changes due to rapid maxillary expansion timing. Angle Orthod., Appleton, v. 75, no. 1, p. 1-6, Jan. 2005.

4. BRESOLIN, D. et al. Mouth breathing in allergic children: Its relationship to dentofacial development. Am. J. Orthod., St. Louis, v. 83, no. 4, p. 334-340, Apr. 1983.

5. CAVALCANTI, M. G. et al. Validation of spiral computed tomography for dental implants. Dentomaxillofac. Radiol. Houndsmills, v. 27, no. 6, p. 329-333, Nov. 1998.

6. CHUNG, C.; FONT, B. Skeletal and dental changes in the sagittal, vertical, and transverse dimensions after rapid palatal expansion. Am. J. Orthod. Dentofacial Orthop., St. Louis, v. 126 , no. 5 , p. 569-575, Nov. 2004.
7. GARRET, B. J. et al. Skeletal effects to the maxilla after rapid maxillary expansion assessed with cone-beam computed tomography. Am. J. Orthod. Dentofacial Orthop., St. Louis, v. 134, no. 1, p. 8e1-8e11, July 2008.

8. HESSE, K. L. et al. Changes in condylar position and occlusion associated with maxillary expansion for correction of functional unilateral posterior crossbite. Am. J. Orthod. Dentofacial Orthop., St. Louis, v. 111, no. 4, p. 410-418, Apr. 1997.

9. INFANTE, P. F. An epidemiological study of finger habits in preschool children, as related to malocclusion, socioeconomic status, race, sex, and size of community. J. Dent. Child., Fulton, v. 43, no. 3, p. 33-38, June 1976.

10. KNOERNSCHILD, K.; AQUILINO, S.; RUPRECHT, A. Transcranial radiography and linear tomography: A comparative study. J. Prosthet. Dent., St. Louis, v. 66, no. 2, p. 239-250, Aug. 1991.

11. KUROL, J.; BERGLUND, L. Longitudinal study and costbenefit analysis of the effect of early treatment of posterior crossbites in the primary dentition. Eur. J. Orthod., Oxford, v. 14 , no. 3, p. 173-179, June 1992. 
12. KUTIN, G. HAWES, R. Posterior crossbites in the deciduous and mixed dentition. Am. J. Orthod., St. Louis, v. 56, no. 5, p. 491-504, Nov. 1969.

13. LAM, P. H.; SADOWSKI, C.; OMERZA, F. Mandibular asymmetry and condylar position in children with unilateral posterior crossbite. Am. J. Orthod. Dentofacial Orthop., St. Louis, v. 115, no. 5, p. 569-575, May 1999.

14. LANGBERG, B.; ARAI, K.; MINER, R. Transverse skeletal and dental asymmetry in adults with unilateral posterior crossbite. Am. J. Orthod. Dentofacial Orthop., St. Louis, v. 127, no. 1, p. 6-16, Jan. 2005.

15. LARSSON, E. The effect of dummy-sucking on the occlusion: A review. Eur. J. Orthod., Oxford, v. 8, no. 2, p. 127-130, May 1986

16. LIMA FILHO, R. M. A.; LIMA, A. C.; RUELLAS, A. C. O. Spontaneous correction of Class II malocclusion after rapid palatal expansion. Angle Orthod., Appleton, v. 73, no. 6, p. 745-752, Dec. 2003

17. LINDER-ARONSON, S. Adenoids: Their effect on mode of breathing and nasal airflow and their relationship to characteristics of the facial skeleton and dentition. Acta Otolaryngol. Suppl., Stockholm, v. 265, p. 1-132, 1970.

18. MELSEN, B.; STENSGAARD, K.; PEDERSEN, J. Sucking habits and their influence on swallowing pattern and prevalence of malocclusion. Eur. J. Orthod., Oxford, v. 1, no. 4, p. 271-280, 1979.

19. MONGINI, F. The importance of radiography in the diagnosis of TMJ dysfunctions: A comparative evaluation of transcranial radiographs and serial tomography. J. Prosthet. Dent. St. Louis, v. 45, no. 2, p. 186-198, Feb. 1981.

20. MYERS, D. R. et al. Condilar position in children with functional posterior crossbite before and after crossbite correction. Pediatr. Dent., Chicago, v. 2, no. 3, p. 190-194, Apr. 1980

21. PHATOUROS, A.; GOONEWARDENE, M. S. Morphologic changes of the palate after rapid maxillary expansion: A 3-dimensional computed tomography evaluation. Am. J. Orthod. Dentofacial Orthop., St. Louis, v. 134, no. 1, p. 117-124, July 2008.

22. PINTO, A. S. et al. Morphological and positional asymmetries of young children with functional unilateral posterior crossbite. Am. J. Orthod. Dentofacial Orthop., St. Louis, v. 120, no. 5, p. 513-520, Nov. 2001.

23. PIRTTINIEMI, P. et al. Relationships of bicondylar position to occlusal asymmetry. Eur. J. Orthod., Oxford, v. 13, no. 6, p. 441-446, Dec. 1991
24. PULLINGER, A. G. et al. Relationship of mandibular condylar position to dental occlusion factors in an asymptomatic population. Am. J. Orthod. Dentofacial Orthop., St. Louis, v. 91, no. 3, p. 200-206, Mar. 1987.

25. RECK, S. F.; FIELDING, A. F. Computed tomography in temporomandibular joint diagnosis. N. Y. State Dent. J., New York, v. 52, no. 8, p. 37-40, Oct. 1986.

26. RILO, B. et al. Midline shift and lateral guidance angle in adults with unilateral posterior crossbite. Am. J. Orthod. Dentofacial Orthop., St. Louis, v. 133, no. 6, p. 804-808, June 2008.

27. SANDIKCCIOGLU, M.; HAZAR, S. Skeletal and dental changes after maxillary expansion in the mixed dentition. Am. J. Orthod. Dentofacial Orthop., St. Louis, v. 111, no. 3, p. 321-327, Mar. 1997.

28. SARI, Z. et al. Rapid maxillary expansion. Is it better in the mixed or in the permanent dentition? Angle Orthod., Appleton, v. 73, no. 6, p. 654-661, Dec. 2003.

29. SILVA FILHO, O. G.; ALVES, R. M.; CAPELOZZA FILHO, L. Alterações cefalométricas ocorridas na dentadura mista após o uso de um expansor fixo tipo quadrihélice. Ortodontia, São Paulo, v. 19, n. 2, p. 22-33, jul./dez. 1986.

30. SILVA FILHO, O. G.; MONTES, L. A. P.; TORELLY, L. F. Rapid maxillary expansion in the deciduous and mixed dentition evaluated through posteroanterior cephalometric analysis. Am. J. Orthod. Dentofacial Orthop., St. Louis, v. 107, no. 3 p. 268-275, Mar. 1995

31. TANIMOTO, K. et al. Comparison of computed with conventional tomography in the evaluation of temporomandibular joint disease: A study of autopsy specimens. Dentomaxillofac. Radiol., Houndsmills, v. 19, no. 1, p. 21-27, Feb. 1990.

32. THILANDER, B.; WAHLUND, S.; LENNARTSSON, B. The effect of early interceptive treatment in children with posterior crossbite. Eur. J. Orthod., Oxford, v. 6, no. 1, p. 25-34, Feb. 1984.

33. VITRAL, R. W. et al. Computed tomography evaluation of temporomandibular joint alterations in patients with Class II Division 1 subdivision malocclusions: Condyle-fossa relationship. Am. J. Orthod. Dentofacial Orthop., St. Louis, v. 126, no. 1, p. 48-52, July 2004.

34. VITRAL, R. W. et al. Temporomandibular joint alterations after correction of a unilateral posterior crossbite in a mixed-dentition patient: A computed tomography study. Am. J. Orthod. Dentofacial Orthop., St. Louis, v. 132, no. 3, p. 395-399, Sept. 2007.
Endereço para correspondência

Edgard Norões Rodrigues da Matta

Rua Sandoval Arroxelas n ${ }^{\circ} 166$, apto. 802 - Ponta Verde

CEP: 57.035-230 - Maceió / Alagoas

E-mail: edgardmatta@ig.com.br 